\title{
Treating irritable bowel syndrome through an interdisciplinary approach
}

\author{
Dominika Dorota Nelkowska
}

Kazimierz Wielki University, Bydgoszcz, Poland

\begin{abstract}
Irritable bowel syndrome (IBS) is a functional disorder with a multifactorial etiology and a complex clinical picture. The recent discovery of the dysregulation of the gut-brain axis as an important pathogenetic mechanism for the development of IBS is a kind of breakthrough in the understanding of IBS and prevalent comorbidities. Nevertheless, IBS treatment still causes many problems and often turns out to be ineffective or brings only short-term effects in reducing symptom severity. In reference to the characteristics of IBS, including new findings regarding etiopathogenesis, an interdisciplinary treatment approach is proposed and the roles of medical and psychological interventions are underlined. The literature search was conducted using electronic databases with a focus on the latest publications. The review may be useful for matching the best strategy of IBS management.
\end{abstract}

Keywords Gut-brain axis, irritable bowel syndrome, management, psychotherapy, treatment

Ann Gastroenterol 2020; 33 (1): 1-8

\section{Introduction}

Irritable bowel syndrome (IBS) is described as a chronic functional gastrointestinal (GI) disorder because of the lack of underlying damage and the absence of structural changes on endoscopic examination [1,2]. Symptomatic characteristics form the basic diagnostic criteria, primarily abdominal pain associated with defecation, changes in the frequency and/or rhythm of bowel movements, and changes in stool consistency [3]. According to the predominant stool pattern, 4 different IBS subtypes can be distinguished: diarrhea-predominant (IBS-D), constipation-predominant (IBS-C), mixed typed (IBS-M), or unclassified (IBS-U) when stool abnormalities are present, but insufficient to meet the criteria of other IBS subtypes [4,5]. IBS does not cause lifethreatening complications, but reduces the quality of life significantly, impairing daily functioning and contributing to high work absenteeism, hospitality and physician visit rates $[5,6]$. Management of heavy IBS also imposes a financial burden [5,7]. Nowadays, IBS is considered as one of the most common medical disorders encountered by all healthcare

Institute of Psychology, Kazimierz Wielki University, Bydgoszcz, Poland

Conflict of Interest: None

Correspondence to: Dominika Dorota Nelkowska, Poland,

e-mail: domanela@gmail.com

Received 6 August 2019; accepted 15 October 2019;

published online 29 November 2019

DOI: https://doi.org/10.20524/aog.2019.0441 providers and its prevalence in developed countries reaches as much as $20 \%$ of the population [8-10]. Therefore, a significant challenge currently faced by physicians is to introduce an appropriate treatment, which has often been ineffective so far.

\section{Objective}

This review is based on the research and guidelines for IBS management. A literature search was conducted using electronic databases (Google Scholar, EBSCO, Springer, ScienceDirect) with a focus on the latest publications. In reference to the characteristics of IBS, including new findings regarding etiopathogenesis, an interdisciplinary treatment approach is proposed and the role of medical and psychological interventions is underlined. The review may be useful for matching the best strategy for IBS management.

\section{Etiopathogenesis of IBS}

IBS symptoms formerly tended to be disregarded and considered rather as a manifestation of neuroticism, neurosis or an excessive self-concern and seeking for sympathy [11]. Although such psychological characteristics were indeed confirmed in studies, IBS is a more complex disorder and its etiopathogenesis goes beyond conversational mechanisms or somatization $[12,13]$. The multiplicity of assignable causes is also reflected in the diversity of symptoms. In addition to chronic or recurrent pain and discomfort and a 
number of GI complaints, many other clinical symptoms are underlined $[4,14]$. These are presented in Table 1.

Multifactorial etiology and complex symptomatology have been systematically verified and thoroughly explained in the last decades. The etiology has been attributed to excessive reactivity of the hypothalamic-pituitary-adrenal (HPA) axis, visceral hypersensitivity, infectious factors, bile acid malabsorption (in IBS-D), enhanced immunological and neuroimmunological reactivity, genetic susceptibility, as well as psychological and social factors [4,6,9,15-19]. Recently specific attention has been devoted to the dysregulation of the gut-brain axis (GBA) and interactions between stress and gut microbiota $[11,13,20,21]$. Proper functioning of the GBA is considered essential for physiological functioning of the digestive system, whereas the intestinal microflora is an important element of the GBA. The terms microbiota and GBA are already used interchangeably to indicate the mutual interactions with the central nervous system (CNS) [22].

The human body is a host organism for trillions $\left(10^{13}-10^{14}\right)$ of microbes residing in the intestine. Although the majority of the GI tract microbiota is still uncharacterized, it is certain that it consists of a great variety of microbes and is responsible for maintaining physical and mental health [22,23]. Microbiota dysbiosis is considered a basic factor that plays a role in initiating and maintaining IBS, whereas the GBA is regarded as substantial, as it integrates brain and GI functions through a bidirectional neurohumoral communication system [21]. The CNS and the GI microbiota can therefore mediate the intestinal sensitivity, motility, secretion, permeability and mucosal immune activation through their dynamic relationship. Dysregulation of the GBA, linked to the genesis of IBS symptomatology, includes several phenomena, such as an altered luminal milieu (e.g., altered microbiota or small intestinal bacterial overgrowth), disrupted gut barrier integrity, impaired immune activation and communication with the CNS $[11,20,21]$. There are ongoing studies that aim to establish links between specific gut microbiota profiles and particular IBS symptoms, but the associations are still unresolved or require further verification $[21,24]$. So far, it has been proven

Table 1 Dominant symptoms of irritable bowel syndrome

\begin{tabular}{ll}
\hline Gastrointestinal symptoms & Other symptoms \\
\hline Abdominal pain & Headache and dizziness \\
Bloating & Muscle pain \\
Constipation & Back pain \\
Diarrhea & Chronic fatigue \\
$\begin{array}{l}\text { Distension } \\
\text { Change in frequency of bowel } \\
\text { movements }\end{array}$ & Painful sexual intercourses \\
$\begin{array}{l}\text { Change in rhythm of bowel urination } \\
\text { movements }\end{array}$ & Gastrointestinal-specific anxiety \\
$\begin{array}{l}\text { Sensation of incomplete } \\
\text { evacuation }\end{array}$ & Depression \\
\hline \begin{tabular}{l} 
Mucous excretion \\
\hline
\end{tabular}
\end{tabular}

that IBS patients have an altered fecal and colonic mucosal microbiota relative to healthy individuals $[11,25,26]$.

Moreover, the research on the CNS has revealed neurofunctional and neuro-structural differences in the brains of IBS patients compared to healthy individuals; these include changes in brain activity related to the perception of visceral stimuli and the regulation of emotions [13]. CNS disorders are also connected with autonomic nervous system (ANS) malfunction. Disturbances of nerve conduction cause hypersensitivity to stimuli and a hyper-reactive response that eventually induces disturbing visceral sensations and abdominal symptoms $[13,27]$. It should be emphasized that the communication network of the GBA, which embraces the CNS, ANS and the HPA axis, is bidirectional. All the systems interact with each other, so any somatic symptom can trigger an emotional response and vice versa [21]. Links between the ANS and HPA axis in IBS pathophysiology are strongly related to neuroendocrine pathways. Research has demonstrated that IBS patients have significantly elevated levels of endothelin, neuropeptide $\mathrm{Y}$ and serotonin (5-hydroxytryptamine, 5-HT), associated with IBS psychological features, as well as sensory, secretory and motor functions of the intestine [14]. Furthermore, the role of stress seems extremely important for IBS pathophysiology. Altered cardiovascular autonomic reactivity to stress has been detected in IBS patients, related to circulating levels of cortisol-another link between the ANS and HPA axis. Stress (both acute and chronic) adversely affects the gut barrier integrity, leading to qualitative and quantitative changes within the microbiota (bacterial dysbiosis) and the development of the "leaky gut syndrome", causing the exacerbation of symptoms $[11,21]$. Studies have indicated that stress changes the internal environment of the GI tract via immune, neurochemical and physiological mechanisms, and leads to an increase in the number of pathological species of bacteria [20].

\section{IBS management}

The cause of IBS has not been clearly identified so far, so the possibility of an effective cure was also limited [19]. However, the remarkable progress in our knowledge of the pathophysiology of IBS that has been made in recent years allows for a better understanding of this condition and brings new perspectives for IBS management. Despite the growing number of publications on various IBS treatment strategies, there is no universally accepted treatment protocol, while current clinical guidelines often indicate the need for further research or clarification [28]. Considering the complex nature of IBS, treatment should be interdisciplinary according to the biopsychosocial model [12,29]. Assembling a group of different specialists (including gastroenterologists, psychologists, psychiatrists, urologists, gynecologists, etc.) would allow a proper diagnosis and further actions according to the totality of patient complaints. Differences between isolated interventions and a multidisciplinary approach are presented in Fig. 1 and 2.

A multidimensional approach should become an essential part of clinical practice as a "lone ranger" model is no longer sufficient for present-day gastroenterology, or even medicine in general [30]. 


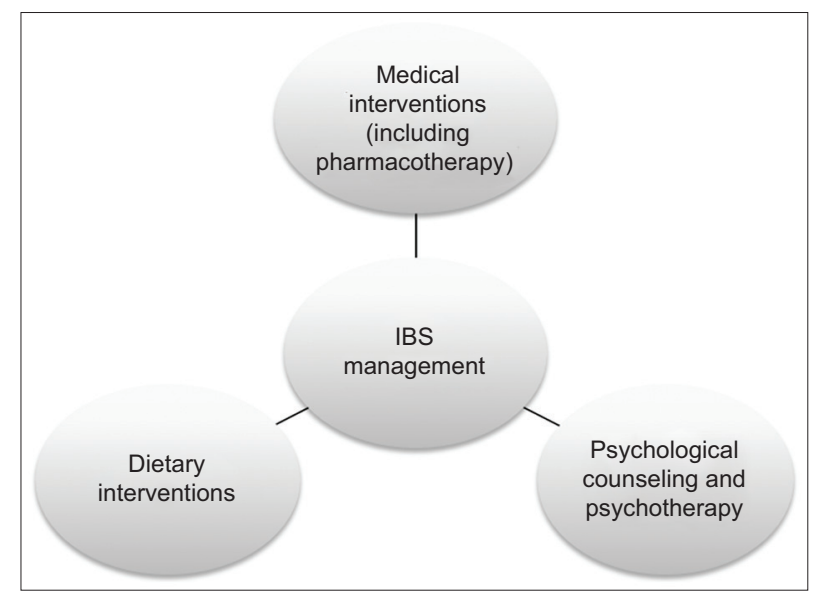

Figure 1 Isolated methods of irritable bowel syndrome management

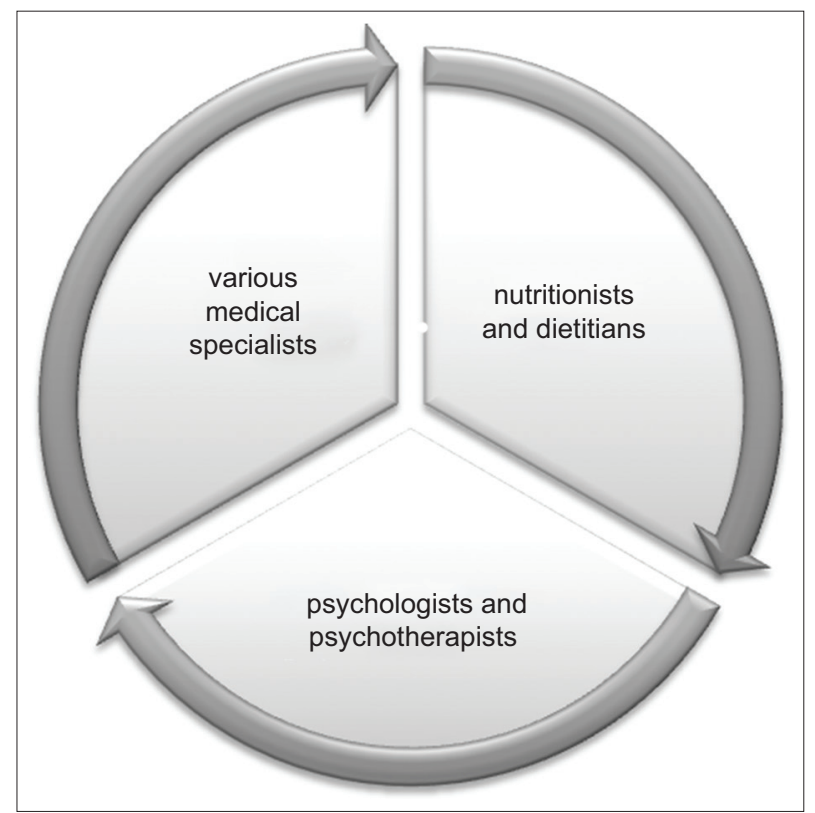

Figure 2 Interdisciplinary team for irritable bowel syndrome management

The diagnostic complexity of IBS is presented in Table 2, showing the possible diagnostic traps awaiting the consultant. Central sensitivity syndromes share common pathophysiological features and overlap; therefore, only a well-knit group of specialists can adequately diagnose and treat functional GI disorders, including IBS [30]. Comprehensive management of IBS should then start with an adequate diagnosis and include both pharmacological and non-pharmacological methods of treatment, according to disease severity and the patient's needs.

\section{Pharmacological management}

Many different drugs have been proposed for IBS treatment, but their real advantages and justification for their use are
Table 2 Brief presentation of the diagnostic complexity of irritable bowel syndrome

\begin{tabular}{|c|c|}
\hline Frequent comorbidities & Differential diagnosis \\
\hline $\begin{array}{l}\text { Gastroesophageal reflux } \\
\text { disease }\end{array}$ & Ulcerative colitis \\
\hline Functional dyspepsia & $\begin{array}{l}\text { Inflammatory bowel disease including } \\
\text { Crohn's disease }\end{array}$ \\
\hline $\begin{array}{l}\text { Chronic fatigue } \\
\text { syndrome }\end{array}$ & Malabsorption syndromes \\
\hline Chronic pelvic pain & Diverticular disease \\
\hline $\begin{array}{l}\text { Temporomandibular } \\
\text { joint disorders }\end{array}$ & Colorectal cancer \\
\hline Fibromyalgia & Endometriosis \\
\hline Eating disorders & Food allergies and food intolerances \\
\hline Affective disorders & Psychiatric disorders \\
\hline
\end{tabular}

being questioned $[16,28,31]$. Considering the complexity of IBS etiopathogenesis and the diversity of prevailing complaints, it is unlikely that drugs acting on a single receptor or pathophysiologic mechanism would provide a significant therapeutic change compared to placebo [16,31]. However, meta-analysis results have already been published in which the examined preparations, particularly alosetron and ramosetron, proved to be more effective than placebo in the treatment of IBS-D and IBS-M [32]. Nevertheless, many pharmacological interventions are only partially effective or cause a wide range of unpleasant side effects $[6,9,31,33]$. Regardless of these contraindications, patients are often treated pharmacologically. Pharmacological management involves mainly laxatives, antidiarrheal and relaxant drugs, agonists and antagonists of the serotonin receptors, or antibiotics $[4,6,34]$. The type of medication depends on the predominant symptom. Treatment of IBS-D includes mainly loperamide, which may decrease the severity of diarrhea, but it is not currently recommended because of a lack of high-quality evidence and no improvement in the overall symptoms $[5,6,13]$. Recently eluxadoline received approval from the US Food and Drug Administration and so it may be an alternative drug for IBS-D $[5,33]$. Eluxadoline is a mixed $\mu$-opioid receptor agonist and $\delta$-opioid receptor antagonist that acts locally on the intestinal nervous system and reduces contractility and secretion in the GI tract $[5,13]$. The efficacy of eluxadoline is sufficient, and it shows significant advantage over placebo in IBS-D; however, it may increase the risk of acute pancreatitis, especially in patients with history of cholecystectomy, pancreatitis, alcohol abuse or liver diseases [13]. Rifaximin, a non-systemic antibiotic, is suggested for IBS-D, IBS-M, and IBS-U treatment $[5,13,24]$. Studies have suggested that it modulates the gut microbiota (affecting mainly harmful bacteria) and contributes to improvements in pain and stool consistency, although the actual benefits depend on the severity of the initial symptoms [5]. IBS-C can be treated with osmotic laxatives, including linaclotide, a guanylate cyclase- $\mathrm{C}$ agonist, which increases the secretion of fluids and accelerates intestinal transit $[13,33]$. Its effectiveness in reducing overall symptoms is proven, but recommendations are not available 
in all countries. Pharmacological management includes also tricyclic antidepressants (TCAs) and selective serotonin reuptake inhibitors (SSRIs), centrally acting drugs that regulate neuronal response. Antidepressants can not only be helpful in reducing abdominal and global IBS symptoms, but may also change the perception of pain and favorably affect comorbid psychoemotional symptoms $[4,13,34]$. Nevertheless, the efficacy of TCAs and SSRIs often does not surpass that of other drugs [35].

Recently, the popularity of probiotic treatment has been increasing, although there is still conflicting evidence regarding the effects of that method [5,21,36-39]. The efficacy of several single strains has been investigated, including Bifidobacterium infantis, Saccharomyces boulardii, Escherichia coli, Lactobacillus casei or Lactobacillus acidophilus [13]. The results are inconclusive and it is difficult to determine unequivocally whether probiotics really show an advantage over placebo, while the efficacy of specific preparations remains controversial $[13,36,38]$. However, the combination of Lactobacillus and Bifidobacteria seems promising, as studies have confirmed its role in restoring tight junction barrier integrity, while weakening the HPA axis and autonomic nervous system activity $[21,40]$. The main advantage of probiotic supplementation in general is treatment safety and the rarity of side effects [28]. However, there are no quality-control standards and caution is recommended, as the compositions of probiotic supplements vary greatly [5].

Latest reports also indicate the role of vitamin D supplementation [41-43]. Vitamin D is an immune modulator, anti-inflammatory and anti-microbial agent, and has a role in regulating the gut microbiome and controlling gut mucosal inflammation $[41,44]$. Studies have implicated a significant vitamin $\mathrm{D}$ deficiency among patients with IBS, and the first empirical reports indicate the efficacy of vitamin D supplementation, which can improve the severity of symptoms and quality of life $[42,43]$.

Another new IBS treatment proposal that appeared as a result of recognizing the importance of gut microflora modulation is fecal microbiota transplantation (FMT). Treatment involving FMT focuses on intestinal flora replacement and modulation through a transplantation of functional bacteria from healthy human feces into the GI tract of patients, where administration involves capsules or enteral suspension $[5,13,45]$. So far, research results do not provide a clearly positive evaluation. FMT may contribute to changes in the gut microbiota, as patients showed an increase in fecal microbial biodiversity after FMT. However, greater symptom relief was observed in the placebo-controlled group than in the FMT group; therefore, no significant advantage for FMT can be inferred [13,46,47]. Besides, the safety of FMT is currently questionable because there is no research assessing the long-term consequences of that method. For now, the risk of potential damage is considered significant. The reason for the overall apprehension is that FMT entails irreversible interference with the microbiota and microbiome and the potential for development of infections, cancer or autoimmune disorders $[5,13]$.

\section{Dietary interventions}

The role of diet in IBS is still ill defined, although specific food types may entail visceral hypersensitivity and aggravate the abdominal symptoms, or even contribute to a disruption in brain-GI tract crosstalk [5]. The microbiota is influenced by the applied diet, which contains substrates for the fermentation of microorganisms. The relationship between diet and composition of the intestinal bacterial flora and bacterial fermentation products can therefore play an important role in the etiology of the disease, directly or indirectly causing IBS symptoms [22]. According to some publications, however, there is not sufficient evidence for diet treatment efficacy and there are conflicting views on dietary recommendations $[5,22,48]$. Discrepancies relate to issues such as fiber supplementation, highly recommended and prescribed for many years, especially for IBS-C. The latest research shows, however, that only soluble fiber is effective (e.g., psyllium), whereas insoluble fiber (e.g., bran) can exacerbate pain and abdominal symptoms $[13,19]$. Flare-ups are usually caused by high-fat foods, raw fruit and vegetables, milk products, drinks containing caffeine, and an excessive supply of artificial sweeteners [19,22]. Therefore, dietary interventions focused on elimination or restrictions were often implemented. Despite the emergence of some indications of the effectiveness of the elimination diet, including a gluten-free or dairy-free diet, there is not sufficient evidence to support such recommendations $[6,13,19]$. In recent years, many publications on the effectiveness of the low fermentable oligo-, di-, monosaccharides and polyol (FODMAP) diet have been published [49-52]. FODMAPs may trigger GI symptoms in IBS patients and diets low in those food components seem to reduce symptom severity via a reduction in fermentation and regulation of passage in the intestines. Studies comparing low-FODMAP diets to other interventions showed no statistically significant differences, so the efficacy of FODMAP restrictions is probably comparable to that of any other types of intervention [13]. However, the problem may lie in the research methodology. A systematic review revealed a very low quality of data in FODMAP studies [53]. There are very few randomized controlled trials (RCTs) and most of the research is unclear. Potential problems concern the high risk of bias, heterogeneity between study designs, a lack of blinding and no long-term follow ups. In order to determine fairly whether a diet is effective, RCTs should be conducted with a double-blinded challenge design and any future diets should be confidential until sufficient data have been collected [53]. It should also be remembered that patients may have different sensitivities to diets. Therefore, it would be necessary to evaluate safety concerns, especially given that the microbiome may be adversely impacted by exclusion diets. Interestingly, a reduction in symptoms through diet was actually observed in the case of an individually modified diet, aligned together with the patient $[6,22]$. This phenomenon may indicate the patient's psychological need to be "looked after", rather than the presence of a digestive disorder, and explains the overall difficulties in establishing the relevance and efficacy of particular food components. Nonetheless, research into the 
effectiveness of particular diet interventions certainly requires further verification.

\section{Psychological assistance and psychotherapy}

Mind-body co-emergence is no longer a matter of academic theoretical debate, but is being empirically supported by current research. Thanks to the remarkable progress in our knowledge that has been made in recent years, it becomes more understandable that numerous parenteral symptoms in IBS result from disturbances of GBA activity. Not surprisingly then, the risk of developing IBS is higher in lonely and unemployed people, those suffering from depression and those experiencing severe stress as a result of physical and sexual violence $[9,13]$. Moreover, IBS patients report generally more frequent aggravations and a greater stress impact than controls [54], while stressful events precede GI exacerbations in $50-80 \%$ of cases [13]. Because of abnormal pain processing in the CNS, IBS patients have much lower pain tolerance and demonstrate greater activation of emotional arousal networks than healthy controls in response to visceral stimulation [29]. Previous studies based on psychological functioning indicate that patients with IBS report separation anxiety symptoms in childhood and are characterized by an insecure attachment style $[21,55]$. They tend to have high levels of state and trait anxiety [56], visceral anxiety [57], catastrophizing and somatization, depression and alexithymia [57-60], as well as insufficient emotional resistance and regulation [56], insufficient levels of resiliency [61], and a high need for social support [62]. They also display cognitive, affective and behavioral reactions resulting from the fear of emerging symptoms from the digestive system, which has been described as GI-specific anxiety [63]. Most IBS patients manifest psychoform symptoms or suffer from comorbid psychiatric disorders, including anxiety disorders (e.g., generalized anxiety disorder, panic disorder), sleep disturbances, eating disorders, depression or posttraumatic stress disorder $[4,12,16,57,63]$.

Those findings regarding the personality of IBS patients, characterized by social and emotional insecurity and somatization, indicate the importance of psychological and psychotherapeutic interventions in IBS management. Besides, since stress and psychological factors can directly affect gut functioning and contribute to IBS symptoms, interventions based on strengthening self-regulatory mechanisms and effective stress management can be truly relevant for the overall functioning in this population [29]. In particular, GI symptoms can be the consequence of inadequate coping strategies and a cause of individually perceived stress at the same time, forming a vicious circle of symptoms (Fig. 3).

So far, the number of studies confirming the efficacy of psychotherapy in IBS treatment show that this type of intervention leads to an improvement in patients' quality of life, reducing pain and anxiety as well as somatic symptoms, although it is not certain whether specific or non-specific factors are relevant [64-68]. Still, cognitive behavioral therapy (CBT), as well as mindfulness therapy, acceptance and commitment therapy, body awareness therapy and intestinefocused hypnotherapy have been positively verified and proved to be an effective treatment in IBS $[4,6,64,65,68-71]$.

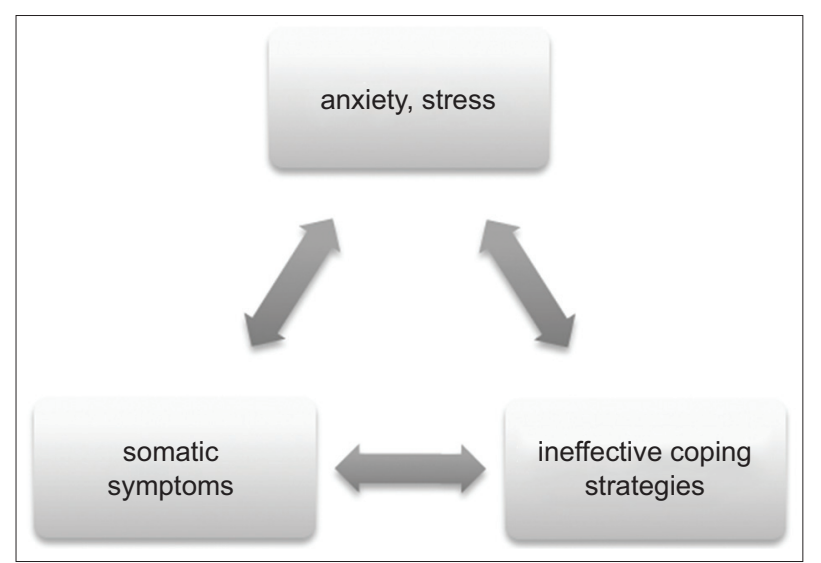

Figure $3 \mathrm{~A}$ vicious circle showing the interrelationships between stress and emotional symptoms and somatic reactions

The most extensive research involves CBT treatments $[29,67,72]$. CBT focuses on modifying behavior and changing dysfunctional thinking patterns to influence mood and physiological symptoms. It includes psychoeducation, relaxation strategies (e.g., breathing exercises), cognitive restructuring (identifying and changing cognitive distortions to generate more accurate and balanced perspectives regarding stress and symptoms), coping skills training and exposure techniques (facing situations the patient is avoiding because of fear of symptoms) [29]. In view of the limited healthcare resources and its high costs and increasing demand, online or internet-delivered CBT approaches have been proposed. Generally the use of online/internet based therapies is controversial, but according to their supporters, such interventions can overcome many existing barriers and provide at least short-term effectiveness in managing depression and anxiety $[73,74]$. However, a systematic review has revealed no sufficient evidence for the effectiveness of online CBT in GI disorders, including IBS [75]. In order to reduce the expenses of psychotherapeutic interventions, programs of selfadministered management are also being introduced [35,76-78]. They usually refer to minimal contact CBT and include selfstudy materials. There is evidence supporting self-management interventions for short-term symptom relief, but long-term outcomes are variable [77,78].

Another quite different proposal refers to psychodynamic therapies, which can be very helpful and effective, although they have not been tested as rigorously in the IBS population as the aforementioned methods-mainly because of the nature of these therapies [67]. Psychodynamic approaches are sometimes perceived as insufficiently "evidence based" and lacking empirical support, but such a statement does not agree with available scientific evidence and may indicate selective dissemination of research results [79-81]. Psychodynamic therapy places emphasis on face-to-face contact and psychotherapeutic alliance, and stands out for its orientation towards affect and expression of emotions, identification and exploration of recurrent thoughts and patterns of functioning, as well as the focus on interpersonal relations; it tends to bring long-lasting effects that persist after treatment [79]. It is a more comprehensive approach and hence may be truly relevant for IBS patients. So far, short-term psychodynamic psychotherapy has 
been empirically supported as a form of IBS treatment $[82,83]$. Specific help with interpersonal problems, followed by psychodynamic interpersonal therapy, proved to improve the health status of patients with severe IBS [84]. Psychodynamic therapies should, however, be given more attention, including short-term and long-term psychotherapies, as these approaches thoroughly explain the psychosomatic perspective in reference to psychoanalytic theories. According to the concepts of many psychoanalysts, along with Joyce McDougall [85], intestinal discomforts may be a bodily manifestation of emotional discomfort, reflecting the psychological determinants of an organic disorder. A consideration of symbolic meanings may be significant for the cognition of the individual emotional patterns that contribute to the development of the disorder [86].

Moreover, as reported by gastroenterologists (information obtained through personal communication, February-March 2018), patients suffering from IBS are often helpless and seeking for support and understanding. A somatic symptom can therefore be a kind of pretext to gain interest. Adopting the role of the patient paradoxically allows a number of psychological benefits to be drawn from the doctor-patient relationship [87]. Besides, positive experiences in interpersonal relations may reduce the symptoms, whereas negative aspects of relationships increase the illness burden of IBS [88]. Creating positive relationships with IBS patients is therefore an important component of the overall care experience. Regardless of the quality of interpersonal relationships in the patient's life, a therapeutic relationship can bring many corrective experiences and help the patient cope with that chronic disorder [19]. In particular, difficulties in the field of psychological and social functioning contribute to a decrease in the quality of life of people with IBS, more than the somatic ailment itself [61]. Therefore, treatment based on symptom attenuation cannot be the only and definitive therapeutic strategy, because IBS patients require a multifaceted approach and psychological support should become one of its inherent elements. Nevertheless, psychological or psychotherapeutic interventions are rarely implemented. In fact, no kind of psychotherapy is routinely recommended for patients with IBS, unless symptoms are severe and incapacitating [4,29]. It is similar in the case of dietary interventions. A survey conducted in the United States has revealed that the majority of gastroenterologists perceive the merit of diet in IBS treatment and believe that patients relate their GI symptoms to eating meals, but only a minority of them refer their patients to registered dieticians [89]. Nonetheless, an interdisciplinary management of IBS seems necessary, regardless of the frequency and intensity of symptoms, especially if any treatment of IBS often takes the form of laborious approach, causing long-term difficulties.

\section{Concluding remarks}

IBS is a disease with a multifactorial etiology, whose major pathogenetic mechanism is a dysregulation of the GBA. Its symptomatology includes mainly GI disorders, resulting from microbiota dysbiosis and visceral hypersensitivity. However, patients also suffer from many parenteral symptoms and often meet the diagnostic criteria for other functional disorders. There is also a substantial prevalence of emotional problems, depression and anxiety, and generally high psychopathology rates. Stress is an important factor contributing to the onset and maintenance of the disorder. Unfortunately, IBS patients have a relatively low level of personal resources and coping skills, which causes significant impediment and entails an increase in symptom severity. With regard to the complex etiology and clinical characteristics of IBS, an interdisciplinary approach should be implemented at both diagnostic and treatment stages. Although such a view is indeed expressed in the current literature, the pathogenetic model still dominates in medical practice and patients are often treated only from a gastroenterologist's perspective, with no access to other types of treatment. However, the justifiability of pharmacological treatment has raised many concerns due to its unpleasant side effects, incomplete efficacy or the lack of long-term improvement. New methods, including probiotic or vitamin D supplementation, seem promising, but are still not comprehensive. Dietary interventions and FMT are controversial for now and require further research. Considering the importance of psychological factors in IBS etiology and course, as well as the wide prevalence of psychoemotional problems in the IBS population, psychological and psychotherapeutic interventions may be truly relevant. Studies have indicated that positive aspects of the therapeutic relationship may contribute to a decrease in the level of stress, depression, anxiety and severity of symptoms. In fact, psychotherapy and psychological interventions are effective in reducing abdominal symptoms and improving the overall quality of life, wherein long-lasting improvement has been demonstrated. Therefore, it seems that psychological help should become an essential element of everyday clinical practice in the treatment of IBS. Minimal contact therapies and self-administrated management should be continually examined in view of the high cost of psychotherapy, which can be a significant burden.

All things considered, systematic healthcare changes need to be undertaken in order to provide the most effective management strategies to help suffering patients. Future efforts should then focus on improving the access to various types of treatment, including psychotherapy.

\section{References}

1. Hyland ME. The origins of health and disease. Cambridge: Cambridge University Press., 2011.

2. Basnayake C. Treatment of irritable bowel syndrome. Aust Prescr 2018;41:145-149.

3. Mulak A, Smereka A, Paradowski L. Novelties and modifications in the Rome IV criteria. Gastroenterol Klin 2016;8:52-56.

4. Videlock EJ, Chang L. Irritable bowel syndrome. In: Small intestine, large intestine, pancreas. Wrocław: Elsevier Urban \& Partner, 2013. p. 437-447.

5. Lacy BE. Diagnosis and treatment of diarrhea-predominant irritable bowel syndrome. Int J Gen Med 2016;9:7-17.

6. Żelowski A, Wojtuń S, Gil J, Dyrla P. Irritable bowel syndrome - diagnostics and treatment principles. Pediatr Med $\operatorname{Rodz}$ 2013;9:250-255. 
7. Jung HK, Kim YH, Park JY, et al. Estimating the burden of irritable bowel syndrome: analysis of a nationwide Korean database. J Neurogastroenterol Motil 2014;20:242-252.

8. Chang L, Lacy BE, Spiegel BM. An evidence-based approach to therapy in IBS-D: a case study compendium. Gastroenterol Hepatol (N Y) 2010;6(9 Suppl 15):1-12.

9. Nehring P, Mrozikiewicz-Rakowska B, Krasnodębski P, Karnafel W. Irritable bowel syndrome - a new approach to aetiopathogenesis. Prz Gastroenterol 2011;6:17-22.

10. Bokic T, Storr M, Schicho R. Potential causes and present pharmacotherapy of irritable bowel syndrome: an overview. Pharmacology 2015;96:76-85.

11. Quigley EMM. The gut-brain axis and the microbiome: clues to pathophysiology and opportunities for novel management strategies in irritable bowel syndrome (IBS). J Clin Med 2018;7.

12. Surdea-Blaga T, Baban A, Nedelcu L, Dumitrascu DL. Psychological interventions for irritable bowel syndrome. J Gastrointestin Liver Dis 2016;25:359-366.

13. Pietrzak A, Skrzydło-Radomańska B, Mulak A, et al. Guidelines on the management of irritable bowel syndrome. Prz Gastroenterol 2018;13:259-288.

14. Stasi C, Bellini M, Gambaccini D, et al. Neuroendocrine dysregulation in irritable bowel syndrome patients: a pilot study. J Neurogastroenterol Motil 2017;23:428-434.

15. Fadgyas-Stanculete M, Buga AM, Popa-Wagner A, Dumitrascu DL. The relationship between irritable bowel syndrome and psychiatric disorders: from molecular changes to clinical manifestations. J Mol Psychiatry 2014;2:4.

16. Bellini M, Gambaccini D, Stasi C, Urbano MT, Marchi S, UsaiSatta P. Irritable bowel syndrome: a disease still searching for pathogenesis, diagnosis and therapy. World J Gastroenterol 2014;20:8807-8820.

17. van Tilburg MA, Palsson OS, Whitehead WE. Which psychological factors exacerbate irritable bowel syndrome? Development of a comprehensive model. J Psychosom Res 2013;74:486-492.

18. Slattery SA, Niaz O, Aziz Q, Ford AC, Farmer AD. Systematic review with meta-analysis: the prevalence of bile acid malabsorption in the irritable bowel syndrome with diarrhoea. Aliment Pharmacol Ther 2015;42:3-11.

19. Adrych K. Irritable bowel syndrome in the light of the latest guidelines. Forum Med Rodz 2018;12:224-233.

20. Moloney RD, Johnson AC, O'Mahony SM, Dinan TG, GreenwoodVan Meerveld B, Cryan JF. Stress and the microbiota-gut-brain axis in visceral pain: relevance to irritable bowel syndrome. CNS Neurosci Ther 2016;22:102-117.

21. Raskov H, Burcharth J, Pommergaard HC, Rosenberg J. Irritable bowel syndrome, the microbiota and the gut-brain axis. Gut Microbes 2016;7:365-383.

22. Dworzański T, Fornal R, Koźba Ł, Celiński K, Dworzańska E. The role of intestinal microbiota in irritable bowel syndrome. Postepy Hig Med Dosw 2018;72:215-226.

23. Wołkowicz T, Januszkiewicz A, Szych J. Gut microbiome and its dysbiosis as an important factor influencing the human health condition. Med Dosw Mikrobiol 2014;66:223-235.

24. Foxx-Orenstein AE. New and emerging therapies for the treatment of irritable bowel syndrome: an update for gastroenterologists. Therap Adv Gastroenterol 2016;9:354-375.

25. Rodiño-Janeiro BK, Vicario M, Alonso-Cotoner C, Pascua-García R, Santos J. A review of microbiota and irritable bowel syndrome: future in therapies. Adv Ther 2018;35:289-310.

26. Distrutti E, Monaldi L, Ricci P, Fiorucci S. Gut microbiota role in irritable bowel syndrome: New therapeutic strategies. World $J$ Gastroenterol 2016;22:2219-2241.

27. Mazur M, Furgała A, Jabłoński K, Mach T, Thor P. Autonomic nervous system activity in constipation-predominant irritable bowel syndrome patients. Med Sci Monit 2012;18:493-499.

28. Tiequn B, Guanqun C, Shuo Z. Therapeutic effects of Lactobacillus in treating irritable bowel syndrome: a meta-analysis. Intern Med 2015;54:243-249.

29. Kinsinger SW. Cognitive-behavioral therapy for patients with irritable bowel syndrome: current insights. Psychol Res Behav Manag 2017;10:231-237.

30. Bellini M, Gambaccini D, Bassotti G. Comorbidities in functional gastrointestinal diseases: Do we need a lone ranger or a dream team? Dig Liver Dis 2016;48:562-564.

31. Occhipinti K, Smith JW. Irritable bowel syndrome: a review and update. Clin Colon Rectal Surg 2012;25:46-52.

32. Black CJ, Burr NE, Camilleri M, et al. Efficacy of pharmacological therapies in patients with IBS with diarrhoea or mixed stool pattern: systematic review and network meta-analysis. Gut 2019 [Epub ahead of print]. doi: 10.1136/gutjnl-2018-318160

33. Camilleri M. Medical therapies in the pipeline for irritable bowel syndrome. Gastroenterol Hepatol (N Y) 2017;13:550-552.

34. Bartnik W. New drugs in functional gastrointestinal disorders. Gastroenterol Klin 2010;2:50-55.

35. Kawanishi H, Sekiguchi A, Funaba M, et al. Cognitive behavioral therapy with interoceptive exposure and complementary video materials for irritable bowel syndrome (IBS): protocol for a multicenter randomized controlled trial in Japan. Biopsychosoc Med 2019;13:14.

36. Yuan F, Ni H, Asche CV, Kim M, Walayat S, Ren J. Efficacy of Bifidobacterium infantis 35624 in patients with irritable bowel syndrome: a meta-analysis. Curr Med Res Opin 2017;33:1191-1197.

37. Doron S, Snydman DR. Risk and safety of probiotics. Clin Infect Dis 2015;60 Suppl 2:129-134.

38. Hill C, Sanders ME. Rethinking "probiotics". Gut Microbes 2013;4:269-270.

39. Ritchie ML, Romanuk TN. A meta-analysis of probiotic efficacy for gastrointestinal diseases. PLoS One 2012;7:e34938.

40. Nébot-Vivinus M, Harkat C, Bzioueche $\mathrm{H}$, et al. Multispecies probiotic protects gut barrier function in experimental models. World J Gastroenterol 2014;20:6832-6843.

41. El Amrousy D, Hassan S, El Ashry H, Yousef M, Hodeib H. Vitamin D supplementation in adolescents with irritable bowel syndrome: Is it useful? A randomized controlled trial. Saudi J Gastroenterol 2018;24:109-114.

42. Jalili M, Vahedi H, Poustchi H, Hekmatdoost A. Effects of vitamin D supplementation in patients with irritable bowel syndrome: a randomized, double-blind, placebo-controlled clinical trial. Int $J$ Prev Med 2019;10:16.

43. Khayyat Y, Attar S. Vitamin D deficiency in patients with irritable bowel syndrome: does it exist? Oman Med J 2015;30:115-118.

44. Sun J. Dietary vitamin D, vitamin D receptor, and microbiome. Curr Opin Clin Nutr Metab Care 2018;21:471-474.

45. Wen W, Zhang H, Shen J, Wei L, Shen S. Fecal microbiota transplantation for patients with irritable bowel syndrome. A metaanalysis protocol. Medicine (Baltimore) 2018;97:e12661.

46. Halkjær SI, Christensen AH, Lo BZS, et al. Faecal microbiota transplantation alters gut microbiota in patients with irritable bowel syndrome: results from a randomised, double-blind placebocontrolled study. Gut 2018;67:2107-2115.

47. Xu D, Chen VL, Steiner CA, et al. Efficacy of fecal microbiota transplantation in irritable bowel syndrome: a systematic review and meta-analysis. Am J Gastroenterol 2019;114:1043-1050.

48. Bartnik W, Chojnacki J, Paradowski L, Skrzydło-Radomańska B, Tomecki R. Recommendations on the management of irritable bowel syndrome. Gastroenterol Klin 2009;1:9-17.

49. Rao SS, Yu S, Fedewa A. Systematic review: dietary fibre and FODMAP-restricted diet in the management of constipation and irritable bowel syndrome. Aliment Pharmacol Ther 2015;41:1256-1270.

50. de Roest RH, Dobbs BR, Chapman BA, et al. The low FODMAP diet improves gastrointestinal symptoms in patients with irritable bowel syndrome: a prospective study. Int J Clin Pract 2013;67:895-903.

51. Schumann D, Klose P, Lauche R, Dobos G, Langhorst J, Cramer H. 
Low fermentable, oligo-, di-, mono-saccharides and polyol diet in the treatment of irritable bowel syndrome: A systematic review and meta-analysis. Nutrition 2018;45:24-31.

52. Varjú P, Farkas N, Hegyi P, et al. Low fermentable oligosaccharides, disaccharides, monosaccharides and polyols (FODMAP) diet improves symptoms in adults suffering from irritable bowel syndrome (IBS) compared to standard IBS diet: A meta-analysis of clinical studies. PLoS One 2017;12:e182942.

53. Dionne J, Ford AC, Yuan Y, et al. A systematic review and metaanalysis evaluating the efficacy of a gluten-free diet and a low FODMAPs diet in treating symptoms of irritable bowel syndrome. Am J Gastroenterol 2018;113:1290-1300.

54. Blanchard EB, Lackner JM, Jaccard J, et al. The role of stress in symptom exacerbation among IBS patients. J Psychosom Res 2008;64:119-128.

55. Ben-Israel Y, Shadach E, Levy S, et al. Possible involvement of avoidant attachment style in the relations between adult IBS and reported separation anxiety in childhood. Stress Health 2016;32:463-471.

56. Orzechowska A, Harasiuk A, Talarowska M, Zboralski K, Chojnacki J, Florkowski A. Assessment of selected psychological factors in patients with irritablebowel syndrome or gastroesopheagal reflux disease. Postępy Psychiatr Neurol 2010;19:115-119.

57. Hazlett-Stevens H, Craske MG, Mayer EA, Chang L, Naliboff BD. Prevalence of irritable bowel syndrome among university students: the roles of worry, neuroticism, anxiety sensitivity and visceral anxiety. J Psychosom Res 2003;55:501-505.

58. Nicholl BI, Halder SL, Macfarlane GJ, et al. Psychosocial risk markers for new onset irritable bowel syndrome-results of a large prospective population-based study. Pain 2008;137:147-155.

59. Phillips K, Wright BJ, Kent S. Irritable bowel syndrome and symptom severity: evidence of negative attention bias, diminished vigour, and autonomic dysregulation. J Psychosom Res 2014;77:13-19.

60. Porcelli P, De Carne M, Leandro G. The role of alexithymia and gastrointestinal-specific anxiety as predictors of treatment outcome in irritable bowel syndrome. Compr Psychiatry 2017;73:127-135.

61. Nelkowska DD. Importance of personal resources for the quality of life of patients with irritable bowel syndrome (IBS). J Educ Health Sport 2019;9:442-453.

62. Wrzesińska MA, Kocur J. The assessment of personality traits and coping style level among the patients with functional dyspepsia and irritable bowel syndrome. Psychiatr Pol 2008;42:709-717.

63. Perkins SJ, Keville S, Schmidt U, Chalder T. Eating disorders and irritable bowel syndrome: is there a link? J Psychosom Res 2005;59:57-64.

64. Lackner JM, Mesmer C, Morley S, Dowzer C, Hamilton S. Psychological treatments for irritable bowel syndrome: a systematic review and meta-analysis. J Consult Clin Psychol 2004;72:1100-1113.

65. Ljótsson B, Falk L, Vesterlund AW, et al. Internet-delivered exposure and mindfulness based therapy for irritable bowel syndrome-a randomized controlled trial. Behav Res Ther 2010;48:531-539.

66. Laird KT, Tanner-Smith EE, Russell AC, Hollon SD, Walker LS. Short-term and long-term efficacy of psychological therapies for irritable bowel syndrome: a systematic review and meta-analysis. Clin Gastroenterol Hepatol 2016;14:937-947.

67. Ballou S, Keefer L. Psychological interventions for irritable bowel syndrome and inflammatory bowel diseases. Clin Transl Gastroenterol 2017;8:e214.

68. Tripathi R, Mehrotra S. Irritable bowel syndrome and its psychological management. Ind Psychiatry J 2015;24:91-93.

69. Hunt MG, Moshier S, Milonova M. Brief cognitive-behavioral internet therapy for irritable bowel syndrome. Behav Res Ther 2009;47:797-802.

70. Miller V, Carruthers HR, Morris J, Hasan SS, Archbold S,
Whorwell PJ. Hypnotherapy for irritable bowel syndrome: an audit of one thousand adult patients. Aliment Pharmacol Ther 2015;41:844-855.

71. Eriksson EM, Andrén KI, Kurlberg GK, Eriksson HT. Aspects of the non-pharmacological treatment of irritable bowel syndrome. World J Gastroenterol 2015;21:11439-11449.

72. Windgassen S, Moss-Morris R, Chilcot J, Sibelli A, Goldsmith K, Chalder T. The journey between brain and gut: A systematic review of psychological mechanisms of treatment effect in irritable bowel syndrome. Br J Health Psychol 2017;22:701-736.

73. Webb CA, Rosso IM, Rauch SL. Internet-based cognitive behavioral therapy for depression: current progress \& future directions. Harv Rev Psychiatry 2017;25:114-122.

74. Lee T-Y, Hsieh T-C, Sung H-C, Chen W-L. Internet-delivered cognitive behavior therapy for young Taiwanese female nursing students with irritable bowel syndrome-a cluster randomized controlled trial. Int J Environ Res Public Health 2019;16.

75. Hanlon I, Hewitt C, Bell K, Phillips A, Mikocka-Walus A. Systematic review with meta-analysis: online psychological interventions for mental and physical health outcomes in gastrointestinal disorders including irritable bowel syndrome and inflammatory bowel disease. Aliment Pharmacol Ther 2018;48:244-259.

76. Lackner JM, Jaccard J, Krasner SS, Katz LA, Gudleski GD, Holroyd K. Self-administered cognitive behavior therapy for moderate to severe irritable bowel syndrome: clinical efficacy, tolerability, feasibility. Clin Gastroenterol Hepatol 2008;6:899-906.

77. Cong X, Perry M, Bernier KM, Young EE, Starkweather A. Effects of self-management interventions in patients with irritable bowel syndrome: systematic review. West J Nurs Res 2018;40:1698-1720.

78. Lackner J, Brenner D, Firth R, et al. Self-administered cognitive behavior therapy for IBS: The IBS outcome study (IBSOS) randomized clinical trial: 2017 ACG Governors Award for Excellence in Clinical Research: 422. Am J Gastroenterol 2017;112:S227.

79. Shedler J. The efficacy of psychodynamic psychotherapy. Am Psychol 2010;65:98-109.

80. Leichsenring F. Are psychodynamic and psychoanalytic therapies effective?: A review of empirical data. Int J Psychoanal 2005;86:841-868.

81. Schimmel J. Psychodynamic psychotherapy. In: Healing in Urology. World Scientific 2015, pp. 243-264.

82. Blanchard EB, Scharff L. Psychosocial aspects of assessment and treatment of irritable bowel syndrome in adults and recurrent abdominal pain in children. J Consult Clin Psychol 2002;70:725-738.

83. Naliboff BD, Fresé MP, Rapgay L. Mind/body psychological treatments for irritable bowel syndrome. Evid Based Complement Alternat Med 2008;5:41-50.

84. Hyphantis T, Guthrie E, Tomenson B, Creed F. Psychodynamic interpersonal therapy and improvement in interpersonal difficulties in people with severe irritable bowel syndrome. Pain 2009;145:196-203.

85. McDougall J. Theaters of the body: a psychoanalytic approach to psychosomatic illness. New York: W. W. Norton \& Company, 1989.

86. Broom BC, Booth RJ,SchubertC. Symbolic diseases and „mindbody” co-emergence. A challenge for psychoneuroimmunology. Explore (NY) 2012; 8: 16-25.

87. Włoszczak-Szubzda A, Jarosz MJ. Patient - disease - physician. Importance and roles in communication. Med Ogólna Nauki $O$ Zdrowiu 2011;17:105-110.

88. Lackner JM, Gudleski GD, Firth R, et al. Negative aspects of close relationships are more strongly associated than supportive personal relationships with illness burden of irritable bowel syndrome. J Psychosom Res 2013;74:493-500.

89. Lenhart A, Ferch C, Shaw M, Chey WD. Use of dietary management in irritable bowel syndrome: results of a survey of over 1500 United States Gastroenterologists. J Neurogastroenterol Motil 2018;24:437-451. 